\title{
Influence of CdS Nano Additives on the Thermal Conductivity of Poly(vinyl chloride)/CdS Nanocomposites
}

\author{
Dinesh Patidar, Narendra Sahai Saxena \\ Semi-Conductor \& Polymer Science Laboratory, Department of Physics, University of Rajasthan, Jaipur, India \\ Email: dinupatidar@gmail.com,n_s_saxena@rediffmail.com
}

Received December 8, 2012; revised January 10, 2013; accepted January 24, 2013

\begin{abstract}
PVC/CdS nanocomposites have been prepared by solution casting method taking 2, 4, 6 and $8 \mathrm{wt} \%$ of CdS nanoparticles. PVC/CdS nanocomposites were characterized through TEM measurement. The measurement of effective thermal conductivity of PVC/CdS nanocomposites has also been done using transient plane source (TPS) method. The effects of concentration of CdS nanoparticles and temperature on the effective thermal conductivity of polymer $\mathrm{PVC} / \mathrm{CdS}$ nanocomposites have been studied. The variation of effective thermal conductivity with the concentration of filler particles and temperature has also been discussed in terms of dispersion of filler particles into polymer matrix and phonon scattering mechanism, respectively.
\end{abstract}

Keywords: Polymer Nanocomposite; Effective Thermal Conductivity; Transient Plane Source Method

\section{Introduction}

Nanoparticles of II-VI group semiconducting materials have received much attention due to their unique optical and electronic properties resulting from quantum size effect $[1,2]$. Cadmium sulphide (CdS) is an important semiconducting material of II-VI group which has its potential applications in sensors, photovoltaic devices, light emitting devices etc. because of their optical, photoluminescence and electroluminescence properties [3-9].

The field of dispersing semiconducting nanoparticles within a polymer matrix is of sufficient interest because of the possibility of producing polymer nanoparticle composite based devices which can be processed in required geometries, morphologies and composition with consequent control over the different electro-optical properties. Therefore, it is important from the fundamental and practical point of view to understand the effects of the incorporation of particles on the physical and chemical properties of the polymer. The properties of filled polymer generally depend on size, shape and nature of the filler particles, their concentration as well as the type of their interaction with polymer matrix.

Among the polymers, poly(vinyl chloride) (PVC) is one of the most common and popular thermoplastic polymers having the characteristics like low price, good optical transparency, excellent electric and thermal insulation and good reproducibility, which are used for the production of various agricultural, domestic, pharmaceutical, medical and other devices [10-12]. Studies on synthesis and physical properties of PVC based nanocompo- site have been reported by many researchers such as $\mathrm{PVC} /$ montmorillonite (MMT) [13], $\mathrm{PVC} / \mathrm{ZnO}$ [14], $\mathrm{PVC} /$ $\mathrm{CNT}$ [15,16], $\mathrm{PVC} / \mathrm{CaCO}_{3}$ [17], $\mathrm{PVC} / \mathrm{ZnS}$ [18], $\mathrm{PVC} / \mathrm{Cd}$ ( $\mathrm{ZnO})$ [19], PVC/CdS [20], PVC/organo-clay [21] etc. It has been observed that the incorporation of nanomaterials into PVC improves the thermal stability, glass transition temperature, specific heat and mechanical properties. To the best of our knowledge and also from the literature survey, no effort has been made to investigate the thermal transport properties such as thermal conductivity of PVC based nanocomposites. The knowledge of thermal properties is required prior to any potential application as the environmental and thermal stability of the material should be known to identify the optimal condition for the performance of a device. The thermal conductivity is that thermal property which is required to describe the heat transfer through the material. The effect of temperature on the thermal properties of a polymer nanocomposite is of great practical importance because most polymers are produced at relatively high temperature and have application in a wide temperature range. In view of the above, in the present study thermal conductivity of $\mathrm{PVC} / \mathrm{CdS}$ nanocomposite has been measured using transient plane source (TPS) method. The results of thermal conductivity with the temperature and concentration of CdS nanoparticles have been discussed.

\section{Experimental Details}

\subsection{Synthesis of CdS Nanoparticles}

CdS nanoparticles have been synthesized using cadmium 
acetate and thiourea [22]. $1 \mathrm{M}$ solutions of cadmium acetate and thiourea have been prepared in the dimethylformamide (DMF) respectively. These solutions were mixed thoroughly with the help of magnetic stirrer. Then this mixed solution was heated at $70^{\circ} \mathrm{C}$ for $3 \mathrm{hrs}$. This heated transparent solution gradually turns into yellowish turbidity. After $3 \mathrm{hrs}$, the precipitate of $\mathrm{CdS}$ has been collected by filtering the solution. Then this precipitate was dried in the vacuum of $10^{-2}$ torr for $10 \mathrm{hrs}$. The dried precipitate has been crushed into fine powder using mortar and pestle.

\subsection{Preparation of Polymer Nanocomposites}

The obtained powder of CdS nanoparticles has now been used to prepare $\mathrm{PVC} / \mathrm{CdS}$ nanocomposites by solution casting method $[23,24]$. In this method, firstly PVC has been dissolved in tetrahydrofurane (THF) solution by magnetic stirrer for $2 \mathrm{hrs}$ and then $\mathrm{CdS}$ nanoparticles with different $\mathrm{wt} \%(2,4,6 \& 8)$ have been dispersed into THF solution containing PVC. The obtained solutions have been agitated by ultrasonicator to get the uniform distribution of nanoparticles in the solution. These solutions have been poured in the petri dishes to obtain the $\mathrm{PVC} / \mathrm{CdS}$ nanocomposite films with different $\mathrm{wt} \%$ of $\mathrm{CdS}$. After 2 days, the nanocomposite films have been taken out from the petri dishes and dried in vacuum of $10^{-2}$ torr for $12 \mathrm{hrs}$ to remove the solvent.

\subsection{Transmission Electron Microscope Measurements}

The transmission electron microscopy (TEM) measurements of PVC/CdS nanocomposites have been performed on TECNAI G2 20 U-TWIN system operating at an accelerating voltage of $200 \mathrm{kV}$. The samples for TEM measurement have been prepared by dissolving $\mathrm{PVC} /$ $\mathrm{CdS}$ nanocomposite films in THF solvent using ultrasonicator. A drop of prepared solution was placed on the carbon coated copper grid and solvent removed by evaporation at room temperature.

\subsection{Thermal Conductivity Measurements}

Transient plane source technique has been used to determine the thermal conductivity of films of $\mathrm{PVC} / \mathrm{CdS}$ nanocomposites. Details of TPS technique have been given elsewhere $[25,26]$. For the measurement of thermal conductivity of thin film with thickness of the order of microns, the experiment is performed in two steps. In the first step, TPS sensor (heat source or temperature sensor) is sandwiched between the two samples having smooth surfaces, and then this arrangement is placed between the two auxiliary metal (stainless steel) pieces. Data for the temperature increase over a given time are collected using the Hot Disc Thermal Constant Analyzer. In the sec- ond step, the experiment is repeated with the sensor sandwiched between the same two pieces of the metal and data for the temperature increase are again collected taking the same experimental conditions as mentioned in the first step. Employing these two temperature increase, thickness of the film and power delivered to the sample, the effective thermal conductivity $(\lambda)$ can be determined through the following relation:

$$
\lambda=\frac{P}{2 A} \frac{\Delta x}{\Delta T}
$$

where $P$ is the output of power given to the heat source (sensor), $A$ is the area of conducting pattern of heat source, $\Delta x$ is the thickness of thin samples and $\Delta T$ is the temperature difference across one of the samples. The factor 2 is due to the symmetrical distribution of heat flux on both sides of the sample.

\section{Results and Discussion}

\subsection{Study of Morphology}

Figures 1(a)-(d) show the TEM micrographs of PVC/CdS nanocomposites. From the figure, it is observed that dispersed $\mathrm{CdS}$ nanoparticles create considerable change in morphology of the pure PVC. In PVC/CdS nanocomposites, $2 \mathrm{wt} \%$ of $\mathrm{CdS}$ nanoparticles in $\mathrm{PVC}$ have almost uniform dispersion in the PVC matrix. The average particle size of CdS nanoparticles is $10 \mathrm{~nm}$. The particles are agglomerated at higher weight percentage of $\mathrm{CdS}$ nanoparticles. The agglomeration increases with the increase of $\mathrm{CdS}$ nanoparticles. It is maximum for $8 \mathrm{wt} \% \mathrm{CdS}$ nanoparticles in PVC.

\subsection{Study of Effective Thermal Conductivity}

The variation of effective thermal conductivity of PVC/ $\mathrm{CdS}$ nanocomposites has been studied from room temperature to $110^{\circ} \mathrm{C}$. The experimentally observed value of effective thermal conductivity for PVC at room temperature is $0.08 \mathrm{~W} / \mathrm{m}-\mathrm{K}$. The variation of effective thermal conductivity of $\mathrm{PVC} / \mathrm{CdS}$ nanocomposites with temperature is shown in Figure 2. It is seen that the effective thermal conductivity of the nanocomposites shows a similar trend of increasing almost linearly upto a temperature which is characteristic to each sample and beyond that temperature effective thermal conductivity shows approximately constant but different value for the different samples. This temperature is often called glass transition temperature, $T_{g}$ of the composite. The above mentioned behaviour of effective thermal conductivity is explained on the basis of structural changes occurring in $\mathrm{PVC}$ and PVC/CdS nanocomposites during the heating.

In the amorphous polymers like PVC the thermal conductivity in the low temperature region (in the vicinity of room temperature) is controlled by the variation of 

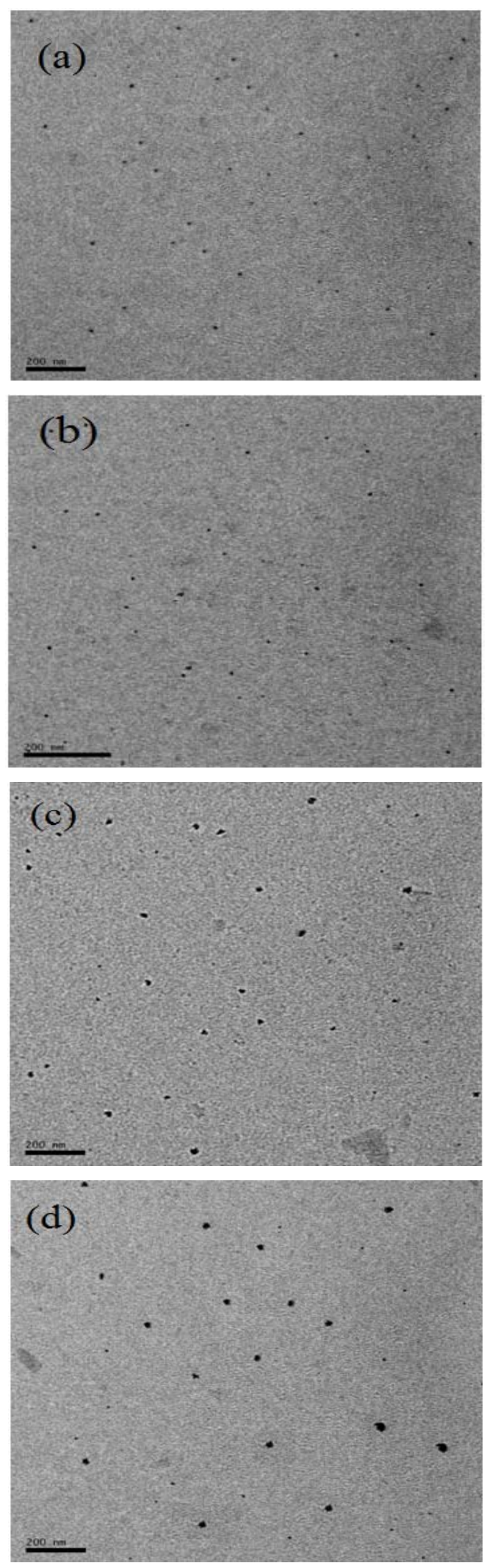

Figure 1. TEM micrographs of PVC/CdS nanocomposites: (a) $2 \%$ CdS, (b) $4 \%$ CdS, (c) $6 \%$ CdS and (d) $8 \%$ CdS.

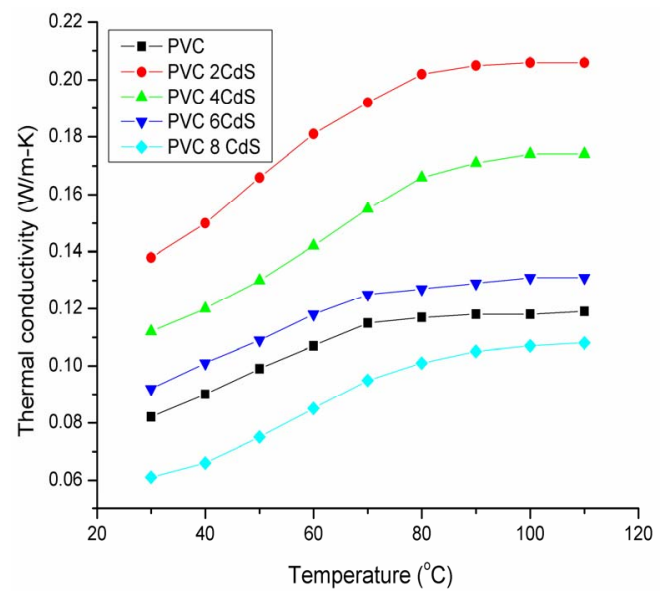

Figure 2. Temperature dependence of effective thermal conductivity of PVC/CdS nanocomposites for different CdS wt\%.

phonon mean free path. For PVC the phonon mean free path is very small [27] because of presence of numerous defects in the amorphous state at room temperature. Certain defects such as bend in chains, gap between two chains, chains of smaller length than others are created in the system during polymerization of the polymers. Therefore in the temperature region (from room to characteristic temperature) the dependence of effective thermal conductivity is controlled $[28,29]$ by the variation of phonon mean free path due to structure scattering and chain defect scattering of the phonons. As the temperature increases from room temperature upto $T_{g}$, the polymer chains straighten out more and more, increasing the corresponding mean free path. This results in the minimization of chain defects and thus the contribution to the corresponding thermal resistance decreases linearly with the rise in temperature and as a result effective thermal conductivity increases almost linearly.

In the temperature range above $T_{g}$, scattering by micro-voids (vacant site scattering) imparts important contribution to the thermal resistance in addition to structure scattering. As the temperature increases above $T_{g}$, polymer transforms gradually from amorphous state to rubbery state. During this transformation, individual polymer units and small chain segments undergo intensive thermal motion producing large torsional rotations and sliding of chain segments. This has two fold effects on the structure of the system: firstly the dominant chain movements create some vacant sites or micro-voids which scatter phonons in the similar way as the point defects; secondly the number and size of these microvoids increase with the increase of temperature. Consequently, the contribution of vacant site scattering to the thermal resistance will increase linearly with temperature and will limit to a constant value.

It is also observed from Figure 2 that the effective 
thermal conductivity of all composites (CdS 2, 4, $6 \mathrm{wt} \%$ ) except with $8 \mathrm{wt} \%$ of CdS nanoparticles is higher than the pure polymer (PVC). This behaviour can be explained on the basis of the structure acquired by the composites in the presence of different concentration of filler nanoparticles of CdS. For lower ( $2 \mathrm{wt} \%$ ) concentration of filler particles a uniform dispersion of nanoparticles is observed in the polymer nanocomposite and a compact structure is acquired resulting into a reduced free volume (nanoparticles acquire the position of voids) and higher effective thermal conductivity in the entire range of temperature [30].

When the filler concentration of CdS nanoparticles increases from $2 \mathrm{wt} \%$ to 4,6 and $8 \mathrm{wt} \%$, a small and increased clustering of CdS nanoparticles is observed in the composites respectively. This change in dispersion increases the particle-particle interaction and decreases the polymer-particle interaction and thus acquiring loose structures as compared to one when CdS filler concentration was $2 \mathrm{wt} \%$. Composite material behaves like two phase material with increasing porosity and hence a decrease in effective thermal conductivity is observed in the whole temperature range of study.

It is interesting to note from Figure 2 that the thermal conductivity of nanocomposite containing $8 \mathrm{wt} \%$ of $\mathrm{CdS}$ into polymer has got the effective thermal conductivity even less than the effective thermal conductivity of pure polymer for all the temperatures under study. This phenomenon is explained [31] by very low efficiency of heat transfer due to interfacial thermal resistance between particles and matrix, so that the higher thermal conductivity of the filler cannot be taken into advantage and the composite behaves like a hollow material, thus reducing its conductivity compared to the dense reference matrix.

\section{Conclusions}

Following conclusions have been drawn from the studies:

- The increase of effective thermal conductivity of PVC/ $\mathrm{CdS}$ nanocomposites increases upto glass transition temperature is attributed to the straightening of the chains and removal of defects. Almost constant value of thermal conductivity beyond glass transition temperature is explained on the basis of structure and vacant site scattering of phonons.

- Thermal conductivity of nanocomposites also increases up to $2 \mathrm{wt} \%$ of the nanoparticles and beyond this $\mathrm{wt} \%$, it decreases, which is due to compact structure of nanocomposites and agglomeration of the CdS nanoparticles, respectively.

\section{Acknowledgements}

N.S. Saxena and D. Patidar thank CSIR and DST, New Delhi respectively, for providing the financial assistance during the course of this work.

\section{REFERENCES}

[1] D. Y. Godovsky, "Device Applications of Polymer Nanocomposites," Advanced Polymer Science, Vol. 153, 2000, pp. 163-205. doi:10.1007/3-540-46414-X_4

[2] M. Chen, Y. Xie, Z. Qiao, Y. Zhu and Y. Qia, "Synthesis of Short CdS Nanofiber/Poly(styrene-altmaleic anhydride) Composites Using Gamma-Irradiation," Journal of Material Chemistry, Vol. 10, No. 2, 2000, pp. 329-332. doi:10.1039/a907743j

[3] Z. L. Wang, "Characterizing the Structure and Properties of Individual Wire-Like Nanoentities," Advanced Materials, Vol. 12, No. 17, 2000, pp. 1295-1298. doi:10.1002/1521-4095(200009)12:17<1295::AID-ADM A1295>3.0.CO;2-B

[4] X. F. Duan, Y. Huang, R. Agarwal and C. M. Lieber, "Single-Nanowire Electrically Driven Lasers," Nature, Vol. 421, No. 6920, 2003, pp. 241-245. doi:10.1038/nature 01353

[5] N. Tokio, F. Keisuke and K. Akio, "High-Efficiency Cadmium-Free $\mathrm{Cu}(\mathrm{In}, \mathrm{Ga}) \mathrm{Se}_{2}$ Thin Film Solar Cells with Chemically Deposited ZnS Buffer Layers," IEEE Transactions on Electronic Devices, Vol. 46, No. 10, 1999, pp. 2093-2097. doi:10.1109/16.792002

[6] V. L. Colvin, M. C. Schlamp and A. P. Alivisatos, "LightEmitting Diodes Made from Cadmium Selenide Nanocrystals and a Semiconducting Polymer," Nature, Vol. 370, No. 6488, 1994, pp. 354-357. doi:10.1038/370354a0

[7] W. U. Huynh, J. J. Dittmer and A. P. Alivisatos, "Hybrid Nanorod-Polymer Solar Cells," Science, Vol. 295, No. 5564, 2002, pp. 2425-2427. doi:10.1126/science. 1069156

[8] J. Ramsden and M. Grutzel, "Photoluminescence of Small Cadmium Sulphide Particles," Journal of Chemical Society: Faraday Transaction, Vol. 80, 1984, pp. 919-933. doi:10.1039/f19848000919

[9] Z. Deng, L. Cao, F. Tang and B. Zou, "A New Route to Zinc-Blende CdSe Nanocrystals: Mechanism and Synthesis," Journal of Physics and Chemistry B, Vol. 109, No. 35, 2005, pp. 16671-16675. doi:10.1021/jp052484x

[10] M. T. F. Arguelles, A. Yakovlev, R. A. Sperling, C. Luccardini, S. Gaillard, A. Sanz Medel, J. M. Mallet, J. C. Brochon, A. Feltz, M. Oheim and W. J. Parak, "Synthesis and Characterization of Polymer-Coated Quantum Dots with Integrated Acceptor Dyes as Fret-Based Nanoprobes," Nano Letters, Vol. 7, No. 9, 2007, pp. 2613- 2617. doi:10.1021/n1070971d

[11] W. H. Starnes Jr., "Structural and Mechanistic Aspects of the Thermal Degradation of Poly(vinyl chloride)," Progress in Polymer Science, Vol. 27, No. 10, 2002, pp. 2133-2170. doi:10.1016/S0079-6700(02)00063-1

[12] M. Sowe, M. Polaskova, I. Kuritka, T. Sedlacek and M. Merchan, "Analysis of Antibacterial Action of Polyvinyl Chloride Surface Modified with Gentian Violet," International Journal of Polymer Analysis and Characterization, Vol. 14, No. 8, 2009, pp. 678-685. doi:10.1080/10236660903298327 
[13] F. Gong, M. Feng, C. Zhao, S. Zhang and M. Yang, "Thermal Properties of Poly(vinyl chloride)/Montmorillonite Nanocomposites," Polymer Degradation and Stability, Vol. 84, No. 2, 2004, pp. 289-294.

doi:10.1016/j.polymdegradstab.2003.11.003

[14] I. S. Elashmawi, N. A. Hakeem, L. K. Marei and F. F. Hanna, "Structure and Performance of $\mathrm{ZnO} / \mathrm{PVC}$ Nanocomposites," Physica B, Vol. 405, No. 19, 2010, pp. 41634169. doi:10.1016/j.physb.2010.07.006

[15] U. Ritter, P. Scharff, T. M. Pinchuk, O. P. Dmytrenko, L. A. Bulavin, M. P. Kulish, Y. I. Prylutskyy, M. A. Zabolotnyy, Y. E. Grabovsky, M. M. Bilyy, A. G. Rugal, A. M. Shut and V. V. Shlapatska, "Radiation Modification of Polyvinyl Chloride Nanocomposites with Multi-Walled Carbon Nanotubes," Material Science and Engineering Technology, Vol. 41, 2010, pp. 675-681.

[16] M. Chipara, J. Cruz, E. R. Vega, J. Alarcon, T. Mion, D. M. Chipara, E. Ibrahim, S. C. Tidrow and D. Hui, "Polyvinylchloride-Single-Walled Carbon Nanotube Composites: Thermal and Spectroscopic Properties," Journal of Nanomaterials, Vol. 2012, 2012, pp. 1-6. doi:10.1155/2012/435412

[17] N. Chen, C. Wan, Y. Zhang and Y. Zhang, "Effect of Nano$\mathrm{CaCO}_{3}$ on Mechanical Properties of PVC and PVC/ Blendex Blend," Polymer Testing, Vol. 23, No. 2, 2004, pp. 169-174. doi:10.1016/S0142-9418(03)00076-X

[18] D. Mitra, I. Chakraborty and S. P. Moulik, "Studies on ZnS Nanoparticles Prepared in Aqueous Sodium Dodecylsulphate (SDS) Micellar Medium," Colloid Journal, Vol. 67, No. 4, 2005, pp. 445-450. doi:10.1007/s10595-005-0117-1

[19] W. E. Mahmoud and A. A. A. Ghamdi, "The Influence of $\mathrm{Cd}(\mathrm{ZnO})$ on the Structure, Optical and Thermal Stabilities of Polyvinyl Chloride Nanocomposites," Polymer Composites, Vol. 32, No. 7, 2011, pp. 1143-1147. doi: $10.1002 /$ pc. 21132

[20] V. Mathur, M. Dixit, K. S. Rathore, N. S. Saxena and K. B. Sharma, "Tensile Study of PVC-CdS Semiconducting Nanocomposite," Optoelectronic and Advanced Material: Rapid Communication, Vol. 3, No. 7, 2009, pp. 685-687.

[21] C. Wan, G. Tian, N. Cui, Y. Zhang and Y. Zhang, "Processing Thermal Stability and Degradation Kinetics of Poly(vinyl chloride)/Montmorillonite Composites," Journal of Applied Polymer Science, Vol. 92, No. 3, 2006, pp. 1521-1526. doi:10.1002/app.20086
[22] D. Patidar, S. Agrawal and N. S. Saxena, "Storage Modulus and Glass Transition Behaviour of CdS/PMMA Nano-Composites," Journal of Experimental Nanoscience, Vol. 6, No. 4, 2011, pp. 441-449. doi:10.1080/17458080.2010.509870

[23] S. Agrawal, D. Patidar and N. S. Saxena, "Glass Transition Temperature and Thermal Stability of ZnS/PMMA Nanocomposites," Phase Transition, Vol. 84, No. 11-12, 2011, pp. 888-900. doi:10.1080/01411594.2011.563152

[24] S. Agrawal, D. Patidar and N. S. Saxena, "Effect of ZnS Nano-Filler and Temperature on Mechanical Properties of Poly(methyl methacrylate)," Journal of Applied Polymer Science, Vol. 123, No. 4, 2012, pp. 2431-2438. doi:10.1002/app. 34800

[25] G. E. Gustafsson, "Transient Plane Source Techniques for Thermal Conductivity and Thermal Diffusivity Measurements of Solid Materials," Review of Scientific and Instruments, Vol. 62, No. 3, 1991, pp. 797-804. doi:10.1063/1.1142087

[26] R. Mangal, N. S. Saxena, M. S. Sreekala, S. Thomas and K. Singh, "Thermal Properties of Pineapple Leaf Fiber Reinforced Composites," Material Science Engineering A, Vol. 339, No. 1-2, 2003, pp. 281-285. doi:10.1016/S0921-5093(02)00166-1

[27] C. L. Choy, "Thermal Conductivity of Polymers," Polymer, Vol. 18, No. 10, 1977, pp. 984-1004. doi:10.1016/0032-3861(77)90002-7

[28] I. I. Perpechoko, "An Introduction to Polymer Physics," Mir, Moscow, 1981.

[29] N. S. Saxena, P. Pradeep, G. Mathew, S. Thomas, M. Gustafsson and S. E. Gustafsson, "Thermal Conductivity of Styrene Butadiene Rubber Compounds with Natural Rubber Prophylactics Waste as Filler," European Polymer Journal, Vol. 35, No. 9, 1999, pp. 1687-1693. doi:10.1016/S0014-3057(98)00247-X

[30] N. S. Saxena, "Thermal and Mechanical Behaviour of Polymer Nanocomposite," Journal of Polymer Engineering, Vol. 30, No. 9, 2010, pp. 575-586. doi:10.1515/POLYENG.2010.30.9.575

[31] Z. Han and A. Fina, "Thermal Conductivity of Carbon Nanotubes and Their Polymer Nanocomposites: A Review," Progress in Polymer Science, Vol. 36, No. 7, 2011, pp. 914-944. doi:10.1016/i.progpolymsci.2010.11.004 